\title{
Die Darstellung der subchondralen Dichtemuster mittels der CT- Osteoabsorptiometrie (CT-OAM) zur Beurteilung der individuellen Gelenkbeanspruchung am Lebenden
}

\author{
M. Müller-Gerbl ${ }^{l}$, R. Putz ${ }^{l}$, N. Hodapp ${ }^{2}$, E. Schulte ${ }^{\prime}$, B. Wimmer ${ }^{3}$ \\ ${ }^{1}$ Anatomische Anstalt München \\ ${ }^{2}$ Abteilung Röntgendiagnostik, \\ ${ }^{3}$ Abteilung Strahlentherapie des Klinikum Freiburg
}

\section{Zusammenfassung}

Aufgrund der Arbeiten von Pauwels und seinen Nachfolgern kann die Verteilung der subchondralen Knochendichte innerhalb einer Gelenkfläche als Meßparameter gelten, der die hauptsächliche Beanspruchung eines Gelenkes widerspiegelt. Das von ihnen angewandte Verfahren der Röntgendensitometrie ist allerdings am Lebenden nicht anwendbar. Unter Benützung der Computertomographie wurde deshalb ein Verfahren entwickelt, die CT-Osteoabsorptiometrie (CT-OAM), die die Dichteverteilungsmuster auch am Lebenden darstellen kann. In einer Vergleichsstudie an Präparaten, die mittels herkömmlicher Röntgendensitometrie und mit der CT-Osteoabsorptiometrie untersucht wurden, zeigte sich, daß das neue Verfahren die gleichen Ergebnisse liefert, allerdings mit dem großen Vorteil, am Lebenden anwendbar zu sein. Weiters wurden die Dichteverteilungsmuster der Cavitas glenoidalis bei Patienten mit verschiedenen Schultererkrankungen und bei Spitzenturnern untersucht. Die Verteilung der subchondralen Knochendichte ergab unterschiedliche Muster bei den verschiedenen Kollektiven. Sowohl der Methodenvergleich als auch die anschließenden Studien zeigen, daß die CT-Osteoabsorptiometrie geeignet ist, Aussagen über die individuelle Beanspruchung eines Gelenkes beim Lebenden zu machen.

\section{Demonstration of Subchrondral Density Patterns by CT Osteoabsorptiometry (CT-OAM) for in Vivo Assessment of Individual Stresses in Joints}

The researches of Pauwels and those following him have demonstrated that the subchondral bone density distribution below the surface of a joint is a metrical parameter which mirrors the predominant stress acting on that joint. Their technique of x-ray densitometry cannot, however, be used during life. By employing computer tomography, a new method has been developed CT-osteoabsorptiometry (CT-OAM) - which can be used to obtain the density distribution pattern in the living subject. By means of a comparative investigation on specimens which were examined both with traditional $\mathrm{x}$-ray densitometry and with CT absorptiometry, it has been shown that the new method can produce the same results, but providing the great advantage to be used on the living. In addition, the density distribution pattern of the glenoid cavity has been examined in patients with various shoulder conditions, and also in gymnasts who exercise on the rings. The distribution of subchondral bone density showed different patterns in the different groups examined. Both the comparison of the methods and the subsequent study confirmed that CT osteoabsorptiometry enables assessment to be made of the individual longterm stresses acting on a living joint.

\section{Einleitung}

Die Größe der lokal einwirkenden Belastung stellt offensichtlich den bestimmenden Faktor bei der Regulation der Knochendichte in den Knochen und Gelenkflächen dar (u.a. Wolff, 1892, Roux, 1912, Pauwels, 1965). Bestimmte sportliche Aktivitäten oder eine Zunah-

Z. Orthop. 128 (1990) 128-133

(C) 1990 F. Enke Verlag Stuttgart me des Körpergewichtes führen zu einer allgemeinen $\mathrm{Zu}$ nahme der Knochendichte (Jones et al., 1977, Nilsson und Westlin, 1971, Chamay und Tschantz, 1972, Woo et al., 1981, Martin et al., 1981), während ein Verlust oder eine Abnahme der körperlichen Aktivität, beispielsweise durch Immobilisation oder längere Bettruhe, zu einer stärkeren Knochenresorption führen (Issekutz et al., 1966, Donaldson et al., 1970, Whedon, 1984). 
Untersuchungen von Pauwels $(1955,1963)$ zeigen, daß sowohl für die Spongiosa als auch für den subchondralen Knochen eine Beziehung zwischen der Verteilung der hauptsächlich vorherrschenden Druckbeanspruchung und der Dichteverteilung besteht. Dieser Zusammenhang ließ ihn von einem ,verkörperten Spannungsfeld" sprechen. Nachfolgende Untersuchungen von Kummer (1962), Knief (1967 a, b), Schmitt (1968), Amtmann und Schmitt (1968) und Amtmann (1971) konnten nachweisen, daß auch eine Korrelation zwischen der Röntgenabsorption als Maß für die Dichte des Knochens und der im spannungsoptischen Modell auftretenden Spannungsverteilung besteht. Es gelang damit der Nachweis des Wolffschen Gesetzes (1892), daß sich der Knochen durch Umbauvorgänge an funktionelle Erfordernisse anpaßt und damit die Verteilung der effektiven Beanspruchung widerspiegelt. Ein von Konermann $(1970,1971)$ entwickeltes photographisches Verfahren zur Bestimmung der Dichteverteilung aus dem Röntgenbild wurde von Schleicher et al. (1980) weiterentwickelt und verfeinert und gilt heute als etablierte Methode der Röntgendensitometrie.

Alle diese Methoden zur Darstellung der Dichteverteilung in der subchondralen Gelenkfläche besitzen aber den Nachteil, daß sie am Lebenden nicht anwendbar sind. Da die Information über die Verteilung der Knochendichte einen hohen diagnostischen Aussagewert in bezug auf die individuelle Belastung und Anpassung eines Gelenkes besitzt, prüften wir, inwieweit und unter welchen Voraussetzungen die Computertomographie eingesetzt werden kann, um die relative Dichteverteilung des subchondralen Knochens zur Darstellung zu bringen.

In einer Vergleichsstudie untersuchten wir deshalb verschiedene anatomische Präparate zunächst mit einem von uns entwickelten CT-absorptiometrischen Verfahren und anschließend mit der herkömmlichen Röntgendensitometrie. Abschließend soll die Anwendung dieses neuen Verfahrens der CT-Osteoabsorptiometrie am Beispiel der Cavitas glenoidalis am Lebenden demonstriert werden.

\section{Material}

1. Anatomisches Untersuchungsgut: 5 Knie-, 3 Sakroiliakal-, 3 Obere Sprung-, 6 Lumbosakralgelenke und 5 Schulterpfannen.

2. Patientengut: CT-Aufnahmen von Schultergelenken von 10 Normalpersonen (Alter: 23-46 Jahre, 6 Männer, 4 Frauen), 10 männlichen Spitzenturnern (Alter: 16-29 Jahre) und 10 Patienten (Alter: 34-56 Jahre, 6 Männer, 4 Frauen) mit Schultererkrankungen.

\section{Methoden}

Zunächst wurden von allen Präparaten CT-Aufnahmen (SOMATOM DR-H, Siemens) mit einer Schichtdicke von $2 \mathrm{~mm}$ angefertigt (Abb. 1a). Anschließend wurden von allen Präparaten $2 \mathrm{~mm}$ dicke Sägeschnitte hergestellt und daran die Röntgendensitometrie durchgeführt. Die CT-Datensätze der Turner und Patienten bestanden ebenfalls aus $2 \mathrm{~mm}$ dicken Schichtaufnahmen.
1. Röntgendensitometrie: Die nicht mazerierten Präparate wurden in planparallele, $2 \mathrm{~mm}$ dicke Scheiben zersägt. Von den $2 \mathrm{~mm}$ dicken Schnitten wurden Röntgenbilder angefertigt, die als Vorlage zur Erzeugung von Äquidensitenbildern dienten, wobei mittels des IBAS 2000 Bereiche gleicher Knochendichte durch die gleiche Farbe bzw. die gleiche Graustufe dargestellt wurden.

Als Fortentwicklung dieser von Schleicher et al. (1980) etablierten Methode wurde eine flächenhafte Darstellung der subchondralen Mineralisierung erreicht, indem die Dichtewerte von den Einzelschnitten in $1 \mathrm{~mm}$ Tiefe gemessen und in eine Flächenkarte übertragen wurden.

2. CT-Osteoabsorptiometrie: Die Dichteverteilung des subchondralen Knochens, ausgedrückt in Hounsfield-Einheiten (HU), wurde in folgendem Verfahren sichtbar gemacht: zur Bestimmung der Isodensiten, d.h. der Linien gleicher Dichte, wurde die Software SIDOS TELE 9.4 (Siemens, Erlangen) verwendet, die zusammen mit einem von Siemens vertriebenen EVADOS Strahlentherapieplanungsrechner läuft. Der CT-Schnitt wird dabei mittels EVA1-Software wie zur Strahlentherapieplanung aufbereitet. Innerhalb der SIDOS TELE-Software wird mittels des Aufrufs „Bereichsgrenzen“ die zur Auffindung von Dichtebereichsgrenzen konzipierte Routine initiiert. Bei Eingabe diverser Hounsfieldwerte erzeugt die Programmroutine Isodensiten, durch die die Regionen größerer Dichte von denen kleinerer getrennt werden (Abb. 1b). Als Dichtebereiche legten wir für alle Schnittbilder fest: 200-399 HU, 400-599 HU, 600-799 HU, 800-999 HU, $<1000 \mathrm{HU}$. Je nach Vergrößerung des Originalschnittes im CT liegt die Auflösung dabei zwischen 0,25-0,8 mm.

Anschließend wurden die so erhaltenen Bilder enner Bildanalyse mit dem Rechner IBAS 2000 (Zeiss, Oberkochen) unterzogen, bei der jeweils gleiche Dichtebereiche mit der gleichen Farbe bzw. den gleichen Grauwerten belegt wurden (Abb. 1c). Eine flächenhafte Darstellung der Dichteverteilung des subchondralen Knochens erreichten wir, indem die Dichtewerte an den Einzelschnitten ebenfalls in einer Tiefe von $1 \mathrm{~mm}$ von der knöchernen Gelenkkontur entfernt gemessen und in eine Dichtekarte der einzelnen Gelenkflächen übertragen wurden (Detaillierte Beschreibung des Verfahrens: Müller-Gerbl et al., 1989).

Dadurch erhielten wir bei beiden Methoden jeweils Dichtekarten der einzelnen Gelenkflächen.

Ergebnisse

Methodenvergleich

a. Stellt man die Falschfarbenbilder vergleichbarer Einzelschnitte - CT-Scan und Röntgenbild - in der gleichen Präparathöhe einander gegenüber, so zeigt sich eine nahezu identische Übereinstimmung der Dichteverteilung, was durch einen systematischen Vergleich der einander entsprechenden Einzelbilder objektiviert werden konnte: $81,7 \%$ der Bildpunkte gleicher Dichte (bei 5 gewählten Dichtestufen) wiesen im Objektbild jeweils dieselben Koordinaten auf. Bei 8,4\% unterschieden sich entsprechende Bildkoordinaten um eine Graustufe, bei $9,9 \%$ um 


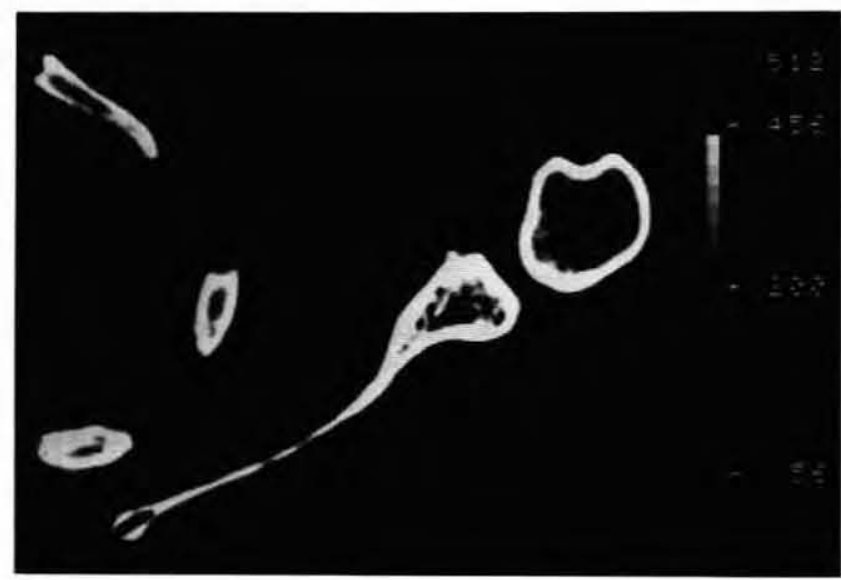

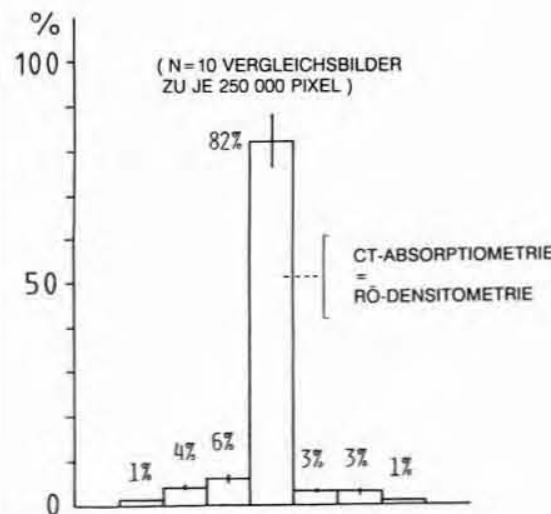

CT-ABSORPT $>$ ROD-DENSI. ROD-DENS. > CT-ABSORPT
Abb. 2 Quantitativer Vergleich zwischen CT-Osteoabsorptiometrie und Röntgendensitometrie: Ermittlung der Bildpunkte (in $\%)$, die bei beiden Verfahren die gleiche Dichtestufen aufweisen, ebenso die Anzahl der Bildpunkte, die sich um eine oder mehrere Stufen unterscheiden
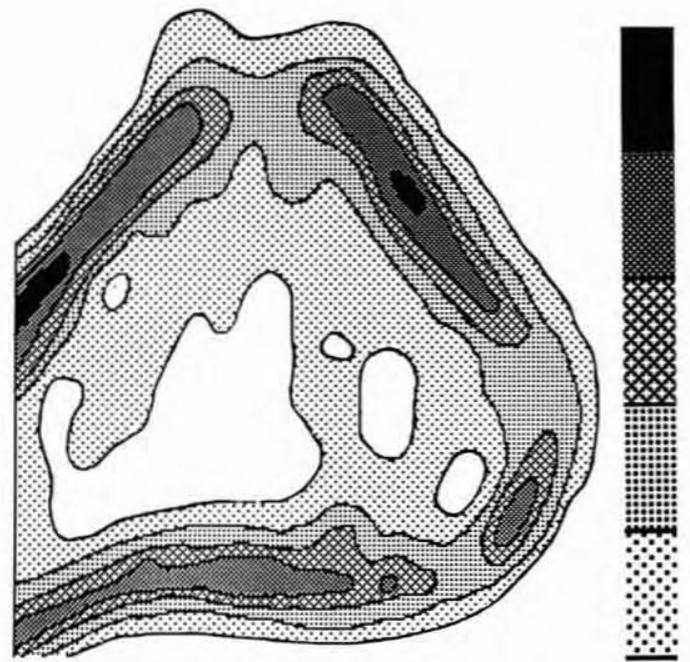

$>1000 \mathrm{HU}$

$800-999 \mathrm{HU}$

$600-799 \mathrm{HU}$

$400 \cdot 599 \mathrm{HU}$

$200-399 \mathrm{HU}$

Abb. 1 CT-Osteoabsorptiometrie eines rechten Schultergelenkes

a) Transversalschnitt

b) Isodensiten im subchondralen Knochen in der Schulterpfanne und im Humeruskopf

c) Schema der verschiedenen Dichtezonen in der Schulterpfanne (Umzeichnung einer Falschfarbendarstellung)
Abb. 3 Flächenhafte Verteilung der subchondralen Mineralisierung in einer Cavitas glenoidalis, Lateralansicht a) Ergebnis mit der Röntgendensitometrie

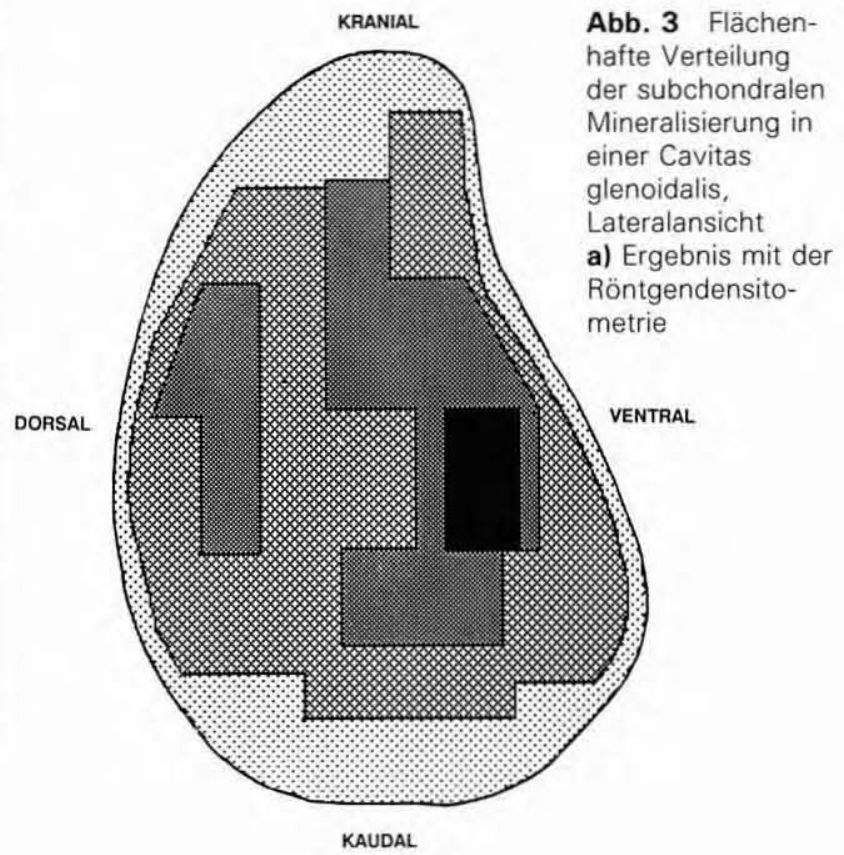

b) Ergebnis mit der CT-Osteoabsorptiometrie 


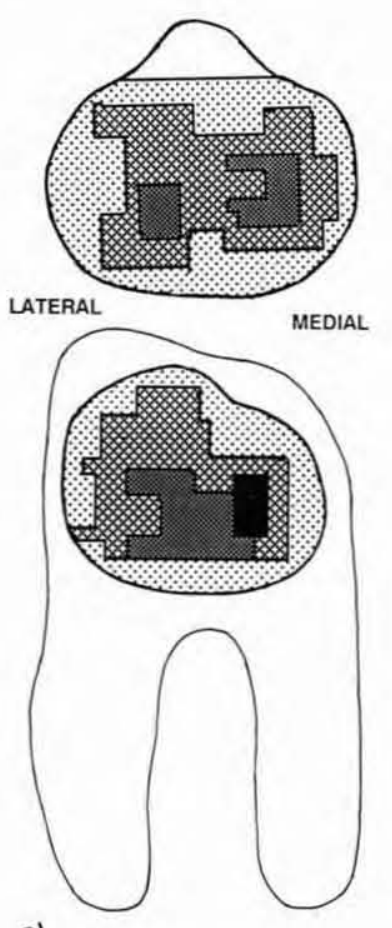

a)

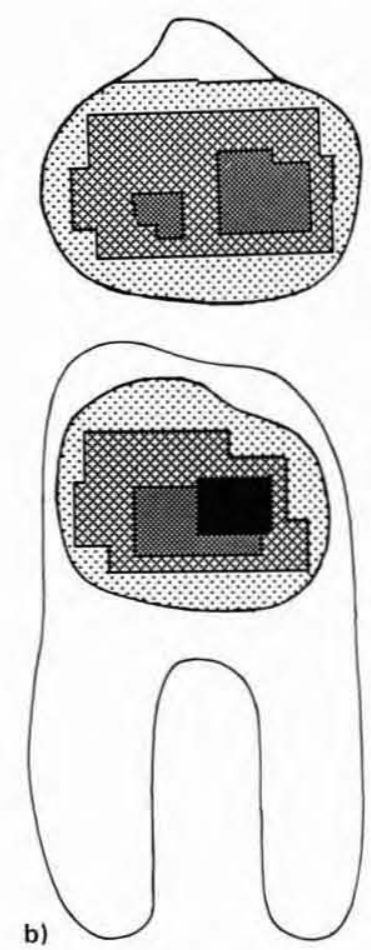

Abb. 4 Flächenhafte Verteilung der subchondralen Mineralisierung in den beteiligten Gelenkflächen des Femoropatellargelenkes (Patella nach oben geklappt)

a) Ergebnis der Röntgendensitometrie

b) Ergebnis der CT-Osteoabsorptiometrie

zwei oder mehr Graustufen. Die entsprechende Histogrammdarstellung zeigt eine Normalverteilung (Abb. 2).

b. Der Vergleich der für eine Gelenkfläche erstellten Dichtekarten aus den Einzelschnitten erlaubt einen direkten und einfachen Vergleich der Ergebnisse beider Methoden. Die Bilder sind kaum voneinander zu unterscheiden, wie die 2 Beispiele in Abb. 3 und 4 demonstrieren.

\section{Subchondrale Dichteverteilungsmuster bei den verschiedenen Kollektiven}

Die Cavitas glenoidalis bei Normalpersonen ohne entsprechende Schmerz- oder Verletzungsanamnese im Schultergelenk (Abb. 5a) weist die höchste Knochendichte immer in den zentralen Anteilen der Schulterpfanne auf, während bei Patienten mit habitueller Schulterluxation oder klinisch relevanter Instabilität abweichende Mineralisierungsmuster mit einer Verschiebung der Dichtemaxima in einem der Randbereiche zu finden sind (Abb. 5b). Bei den Spitzenturnern (Abb. 5c) war die Knochendichte insgesamt in allen Fällen wesentlich höher als bei den beiden anderen Gruppen. Dabei waren die Dichtemaxima zum Teil zentral lokalisiert, zum Teil zum Rand hin verschoben.

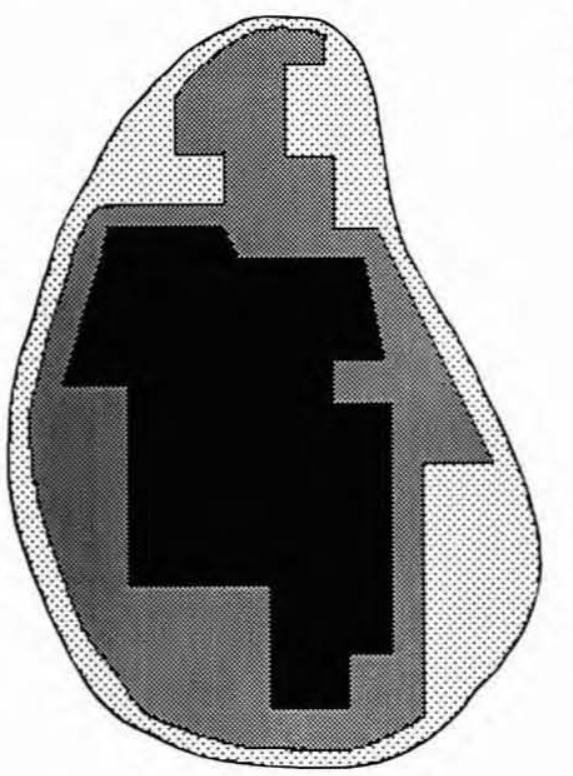

Abb. 5 Dichtekarten der Cavitas glenoidalis bei a) Normalperson

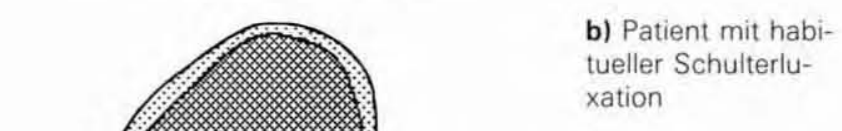
xation

c) Turner

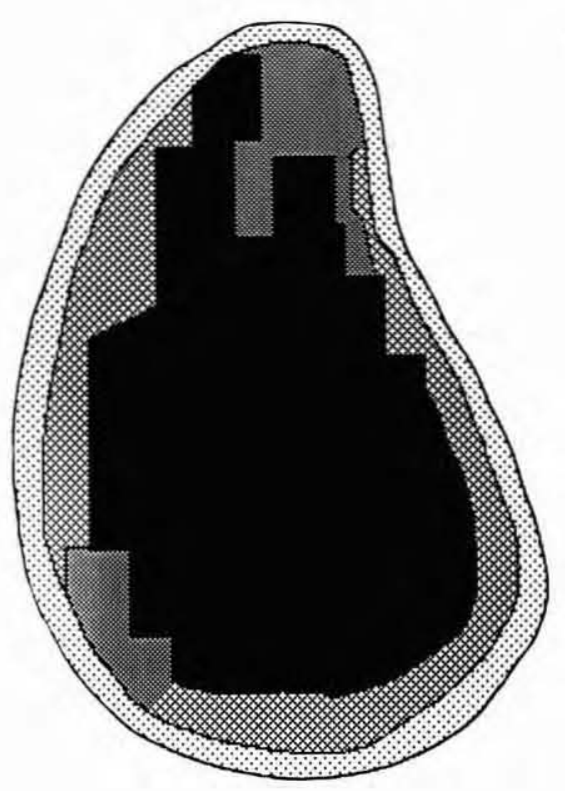




\section{Diskussion}

Unsere Vergleichsstudie an den anatomischen Präparaten ergibt, daß CT-Osteoabsorptiometrie und Röntgendensitometrie zu gleichen Aussagen führen.

Die Tatsache, daß bei einer systematischen Gegenüberstellung vergleichbarer Einzelschnitte, die mit beiden Verfahren ausgewertet wurden, dennoch nur in $81,7 \%$ eine identische Übereinstimmung von Bildpunkten gleicher Dichte vorliegt, ist darauf zurückzuführen, daß die Vergleichsschnitte im allgemeinen geringfügige Lageunterschiede aufweisen, d.h. daß Schnittebene des Präparates und Scanebene nicht exakt in Übereinstimmung zu bringen waren. Bereits bei Einbeziehung der jeweils angrenzenden Dichtebereiche besteht eine Übereinstimmung der beiden Verfahren für $92 \%$ der Bildpunkte.

Damit scheint uns ausreichend gesichert, daß die CT-Absorptiometrie als analytische Methode zur Röntgenabsorptionsmessung am Lebenden eingesetzt werden kann.

Eine wichtige Frage bei der Darstellung dieser Verteilungsmuster liegt darin, ob dabei Veränderungen der Knochendichte insgesamt oder der unterschiedlichen Mineralisierungsgrade nachgewiesen werden. Dazu muß kurz auf die physikalischen Voraussetzungen der CTOsteoabsorptiometrie eingegangen werden. Zur Bestimmung der Dichtestufen wird die Hounsfield-Dichtestufenskala verwendet. Der Hounsfieldwert, der über das CT bestimmt wird, ist ein Maß für den linearen Schwächungskoeffizienten und ist, da er bei den verwendeten Röntgenenergien im wesentlichen vom Photoeffekt abhängt, nur dann ein Maß für die physikalische Dichte, wenn eine gleichartige Verteilung von Elementen verschiedener Ordnungszahlen vorliegt. Da im Knochengewebe der wesentliche Beitrag zur Absorption von den Elementen der höchsten Ordnungszahl, also von den Mineralsalzen abhängt, sind die Hounsfieldwerte in diesem Fall nicht so sehr ein Maß für die physikalische Dichte, sondern ein Maß für die unterschiedliche Mineralsalzkonzentration.

Die flächenhafte Darstellung der Dichteverteilung erlaubt eine schnelle und einfache Beurteilung der individuellen Beanspruchungssituation eines Gelenkes. Zentral lokalisierte Dichtemaxima in der Schulterpfanne, wie sie bei den Normalpersonen in der Cavitas glenoidalis zu finden waren, deuten auf eine gleichmäßige Beanspruchung bei einem zentralen Durchstoßpunkt der Resultierenden hin, während eine Verschiebung der höchsten Dichtewerte in den Randbereich eine auf Dauer exzentrische Lage der Resultierenden und damit eine ungleichmäßige Belastung der Gelenkfläche vermuten läßt. Dadurch werden in den Randbereichen möglicherweise Spitzenspannungswerte erreicht, die auf Dauer zu einer Schädigung des Knorpels und Knochens führen und damit der Entstehung einer Arthrose Vorschub leisten können. Auch Schmerzsyndrome ohne sonstige morphologische Korrelate könnten darin ihre Erklärung finden.

Entsprechend der eingangs erwähnten $\mathrm{Zu-}$ sammenhänge, laufen im Knochen ständig Umbauvorgänge als Anpassungsreaktion an die jeweilige mechanische Situation eines Gelenkes ab. Erhöhte Belastung führt zu ei- ner Dichtezunahme des Knochens (Jones et al., 1977, Nilsson und Westlin, 1971, Chamay und Tschantz, 1972, Woo et al., 1981, Martin et al., 1981), Reduktion der Belastung zur Resorption des Knochens (Issekutz et al., 1966, Donalsson et al., 1970, Whedon, 1984). Dies kann besonders gut durch die Befunde an den Schulterpfannen von Spitzenturnern untermauert werden, die Dichtewerte aufweisen, die deutlich höher liegen als bei Normalpersonen. Gerade vom Ringeturnen ist bekannt, daß in bestimmten Übungsphasen Kräfte bis zum Fünffachen des Körpergewichtes auftreten können, wie u.a. die Untersuchungen von Bodem et al. (1984) zeigen, und damit die hohe Mineralisierung ohne weiteres verständlich machen.

Da sich im Laufe des Lebens die auf die Gelenke einwirkenden Belastungen ändern können, sei es durch eine Aktivitätszunahme oder -abnahme oder durch Zustände, die die Mechanik eines Gelenkes verändern, wie beispielsweise in Fehlstellung verheilte Frakturen oder Umstellungsosteotomien, müssen auch die entsprechenden Dichtemuster in Anpassung an die jeweilige geänderte Situation veränderte Bilder zeigen. Möllers und Mitarbeiter (1986) untersuchten röntgendensitometrisch die Verteilung der subchondralen Knochendichte am distalen Radiusende sowohl bei gesunden Personen als auch bei solchen nach in Fehlstellung verheilten distalen Radiusfrakturen loco typico. Die Materialverteilung in den normalen Radii weist ein typisches Verteilungsmuster auf, das sich erheblich von den Dichteverteilungsmustern, die in den Radii nach in Fehlstellung verheilten Frakturen gefunden wird, unterscheidet. Diese vom Normalbefund abweichenden Muster müssen als Ausdruck einer veränderten hauptsächlichen Spannungsverteilung über die distale Radiusfläche mit einer erhöhten Belastung der dorsalen Abschnitte interpretiert werden und können damit auch die häufig auftretenden Beschwerden mit Gefahr der nachfolgenden Arthrosebildung erklären.

Nach Meniskektomie, sowohl partiell als auch total, ändert sich ebenfalls die Spannungsverteilung im Kniegelenk (Maquet, 1986). Nachfolgend müßten in Anpassung an die neue Situation entsprechende Mineralisierungsmuster auftreten. Dieser Nachweis gelang der Arbeitsgruppe von Odgaard und Mitarbeitern (1988): ein Vergleich der Verteilungsmuster vor und nach dem Eingriff spiegelt die in unterschiedlich hohem Maße erfolgende Spannungszunahme bei Teil- oder Totalentfernung des Meniskus in den Gelenkflächen der proximalen Tibia wider.

Allerdings muß nachdrücklich darauf hingewiesen werden, daß es sich bei der von uns dargestellten Methode nicht um eine Berechnung von absoluten Werten handelt. Es geht vielmehr darum, relative Konzentrationsunterschiede innerhalb einer Gelenkfläche zur Darstellung zu bringen. Zur objektiven, also über die Zeit vergleichbaren, quantifizierten Dichtemessung müßte das Dual-Energie-Verfahren bzw. die Messung mit Referenzphantom benützt werden.

Eine mögliche Korrelation dieser Mineralisierungsmuster mit mechanischen Knocheneigenschaften, wie beispielsweise Festigkeit oder Härte, muß noch geprüft werden. 
Unsere Befunde zeigen, daß die CT-Absorptiometrie ein weites Spektrum an Anwendungsmöglichkeiten bietet. Sie kann zu diagnostischen Zwecken eingesetzt werden, um Aufschluß über die individuelle mechanische Situation eines Gelenkes zu erhalten. Sie kann desweiteren zur Verlaufskontrolle nach Eingriffen oder Traumen eingesetzt werden, die die mechanische Situation eines Gelenkes verändern. Außerdem bietet sie sich zum Einsatz in der klinischen Grundlagenforschung an, da sie eine nicht invasive, den Patienten nicht über Gebühr belastende Untersuchungsmethode darstellt.

Ein weiterer großer Vorteil liegt in der Tatsache, daß gezielt gesunde Kontrollgruppen oder ausgewählte Kollektive, die besonderen Belastungen ausgesetzt sind - seien es bestimmte Sportarten oder verschiedene Berufsgruppen -, damit untersucht werden können.

Damit steht u.E. eine Methode zur Verfügung, die in besonders gelenkbeanspruchenden Bereichen der Sport- und Arbeitsmedizin für die Prävention von Gelenkschäden eingesetzt werden kann, was angesichts der flächendeckenden Zahl von CT-Geräten nicht auf Einzelfälle beschränkt bleiben muß.

Danksagung: Herrn H.-P. Boschert (Abteilung Sporttraumatologie der Universität Freiburg, Vorstand Prof. Dr. A. Klümper) danken wir für die Herstellung von Kontakten mit den Athleten.

\section{Literatur}

1 Amtmann, E.: Mechanical stress, functional adaption and the variation-structure of the femur diaphysis. Ergebn. Anat. Entw.-gesch. Bd. 44, Heft 3, Springer, Berlin-Heidelberg-New York 1971

2 Amtmann, E., H. P. Schmitt: Über die Verteilung der Corticalisdichte im menschlichen Femurschaft und ihre Bedeutung für die Bestimmung der Knochenfestigkeit. Z. Anat. Entw.-gesch. 127 (1968) 25-41

3 Bodem, F., F. Brussatis, W. Menke: Zur theoretischen Biomechanik des Schultergelenkes: Die Entstehung gewöhnlicher und außergewöhnlicher mechanischer Belastungen des glenohumeralen Gelenkknorpels. In: Biomechanik der gesunden und kranken Schulter, Thieme, Stuttgart 1984

${ }^{4}$ Chamay, A., P. Tschantz: Mechanical influences in bone remodelling. Experimental research on Wolff's law. J. Biomechanics 5 (1972) 173-180

5 Donaldson, C., S. Hulley, J. Vogel, R. Hattner, J. Bayers, D. McMillan: Effects of prolonged bed rest on bone mineral. Metabolism. 19 (1970) 1071-1084

${ }^{6}$ Issekutz, B., J. Blizzard, N. Birkhead, K. Rodahl: Effect of prolonged bed rest on urinary calcium output. J. appl. Physiol. 21 (1966) 1013-1020

7 Jones, H., J. Priest, W. Hayes, C. Tichenor, D. Nagel: Humeral hypertrophy in response to exercise. Bone Jt. Surg. 59-A (1977) 204-208
${ }^{8}$ Knief, J.-J.: Materialverteilung und Beanspruchungsverteilung im coxalen Femurende - Densitometrische und spannungsoptische Untersuchungen. Z. Anat. Entw.-gesch. 126 (1967) 81-116

${ }^{9}$ Knief, J.-J.: Quantitative Untersuchung der Verteilung der Hartsubstanzen im Knochen und ihrer Beziehung zur lokalen mechanischen Beanspruchung. Z. Anat. Entw.-gesch. 126 (1967) 55-80

10 Konermann, H.: Dichteverteilung im Röntgenbild des Skeletts. Naturwissenschaften. 57 (1970) 255

11 Konermann, H.: Quantitative Bestimmung der Materialverteilung nach Röntgenbildern des Knochens mit einer neuen photographischen Methode. Z. Anat. 134 (1971) 13-48

12 Kummer, B.: Funktioneller Bau und funktionelle Anpassung des Knochens. Anat. Anz. 111 (1962) 261-293

${ }^{13}$ Maquet, G. J.: Biomechanics of the knee. Springer Verlag, Berlin 1984

${ }^{14}$ Martin, R., J. Albright, W. Clarke, J. Niffenegger: Load-carrying effects on the adult beagle tibia. Sports Exer. 13 (1980) 343-349

15 Möllers, N., K. Lehmann, J. Koebke: Die Verteilung des subchondralen Knochenmaterials an der distalen Gelenkfläche des Radius. Anat. Anz. 161 (1986) 151

${ }^{16}$ Müller-Gerbl, M., R. Putz, N. Hodapp, E. Schulte, B. Wimmer: Computed tomography-osteoabsorptiometry for assessing the density distribution of subchondral bone as a measure of long-term mechanical adaptation in individual joints. Skeletal Radiol. (1989) in press

17 Nilsson, B., N. Westlin: Bone density in athletes. Clin. Orthop. 77 (1971) 179-182

18 Odgaard, A., C. M. Pedersen, S. M. Bentzen, J. Jorgensen, I. Hvid: Density changes at the proximal tibia after menisectomy. 6th Meeting of the European Society of Biomechanics, Bristol 1988

19 Pauwels, F.: Die Druckverteilung im Ellbogengelenk, nebst grundsätzlichen Bemerkungen über den Gelenkdruck. 11. Beitrag zur funktionellen Anatomie und kausalen Morphologie des Stützapparates. Z. Anat. Entw.-gesch. 123 (1963) 643-667

${ }^{20}$ Pauwels, F.: Gesammelte Abhandlungen zur Biomechanik des Bewegungsapparates. Springer, Berlin-Heidelberg-New York 1965

21 Pauwels, F.: Über die Verteilung der Spongiosadichte im coxalen Femurende und ihre Bedeutung für die Lehre vom funktionellen Bau des menschlichen Knochens. 7. Beitrag zur funktionellen Anatomie und kausalen Morphologie des Stützapparates. Morph. Jb. 95 (1955) 35-54

22 Roux, W.: Gesammelte Abhandlungen über Entwicklungsmechanik der Organismen I und II. Engelmann, Leipzig 1895

${ }^{23}$ Schleicher, A., B. Tillmann, K. Zilles: Quantitative analysis of x-ray images with a television image analyser. Microscopia Acta. 83 (1980) 189-196

${ }^{24}$ Schmitt, H. P.: Über die Beziehung zwischen Dichte und Festigkeit des Knochens am Beispiel des menschlichen Femur. Z. Anat. Entw.-gesch. 127 (1968) 1-24

25 Whedon, D.: Dissue osteoporosis: physiologic aspects. Calcif. Tissue Int. 36 (1984) 146-150

26 Wolff, J.: Das Gesetz der Transformation der Knochen. Verlag A. Hirschwald, Berlin 1892

27 Woo, S., S. Kuei, D. Amiel, M. Gomez, W. Hayes, F. White, W. Akeson: The effect of prolonged physical training on the properties of long bone: a study of Wolff's law. J. Bone Jt. Surg. 63-A (1981) 780-787

Dr. M. Müller-Gerbl

Anatomische Anstalt

Pettenkoferstr. 11

8000 München 2 